\title{
Spatial maps in olfactory cortex during olfactory navigation
}

Authors: Cindy Poo (1), Gautam Agarwal (2), Niccolò Bonacchi (1), Zachary Mainen (1)

(1) Champalimaud Foundation, Lisbon, Portugal.

(2) Redwood Center for Theoretical Neuroscience, University of California, Berkeley, CA, USA

\begin{abstract}
:
Animals rely on chemical signals to forage for food, shelter, and mates. Such behaviors call for odor cues to be linked to locations within their environment. Nonetheless, where and how this happens in the brain is not known. Here, we show that spatial and olfactory information converge in posterior piriform cortex (pPCx), an olfactory region with strong associative circuity. Ensembles of pPCx neurons recorded in rats performing an odor-cued spatial navigation task were robustly selective for both odor identity and spatial location, forming an "odor-place map". Spatially-selective pPCx neurons displayed joint selectivity for odors, stability across behavioral contexts and functional coupling to the hippocampal theta rhythm. These results implicate the pPCx as a strong candidate region to associate spatial and olfactory information in support of navigational behavior.
\end{abstract}




\section{Main Text:}

Navigation requires the flexible integration of sensory information with a cognitive map of the environment (1). The hippocampal formation and the primary olfactory (piriform) cortex (PCx) are believed to be dedicated to spatial navigation and olfactory discrimination, respectively (2-8). PCx neurons receive direct sensory input from olfactory bulb (OB) projection neurons $(9,10)$, but also contain dense recurrent olfactory circuitry and receive long-range corticocortical input from regions including the frontal cortex and temporal lobe $(11,12)$, including the hippocampus and the lateral entorhinal cortex (LEC) (12-15). Computational modeling suggests that PCx neural architecture is well-suited to perform associative functions (16-18), which is supported by evidence for odor recognition and memory in PCx (19-22).

While these properties of PCx make it a strong candidate to associate odor and spatial information, there is yet no physiological evidence for spatial representations here. To investigate, we developed a novel odor-cued spatial task that challenged rats to use spatial information along with odor identity to navigate to a specific location for reward. We recorded from the posterior PCx (pPCx) and area CA1 of the hippocampus in rats performing this task. We focused on pPCx due to the relative preponderance of associative circuits and connectivity to higher-order regions that have been found there $(12,21-24)$. Our behavioral arena consisted of an elevated plus-maze with a 1 square meter footprint that was positioned in a room with visual landmarks (Fig. 1A and supplementary video). At the end of each arm was a port, each of which could deliver either an odor stimulus or a water reward.

Trials began when an LED was activated at one of the four ports. If the rat inserted its snout into the lighted port, it received one of four odors chosen at random. Each odor could be presented at any one of the four possible initiation ports and, no matter where it was presented, it indicated a fixed reward location (e.g. odor 1 the north, odor 2 to the south, etc.) (Fig. 1A, B). 
After sampling the odor, the animal could receive a water reward by navigating to the appropriate reward port (Fig. S1). Odors and initiation port locations were randomly interleaved across trials, such that the proportion of trials was balanced across both variables. This combination of initiation and goal ports incentivized rats to use an allocentric (world-centered) navigational strategy, since this would be simpler than the alternative of learning and applying a different egocentric (body-centered) rule for each odor depending on where it was sampled (25, 26). Rats showed a bias to remain in the same arm where the odor was presented (Fig. S1C, 'stay'), but performance was otherwise similar across odors, choice locations and directions (Fig. 1E and F). To test whether rats used an allocentric strategy, we analyzed the history dependence of choices for evidence of "win-stay" behavior (see Methods). We found that rats indeed showed a allocentric bias to revisit the port that in the previous trial was rewarded (north, south, etc.), but no egocentric bias to repeat the same action (left, right, etc.) (Fig. 1C, D, 6 rats, $\sim 40,000$ trials).

After training (criterion: $>70 \%$ accuracy, chance level $=25 \%,>200$ trials/session), rats were implanted with a nasal thermocouple to measure sniffing $(6,27)$ and a microdrive with 24 independently-moveable tetrodes; 19 of these targeted pPCx and 5 targeted dorsal CA1 area of the hippocampus. We obtained 995 well-isolated units (neurons) from pPCx and 154 from CA1 in 3 rats across 44 total sessions (Fig. 1, E and F and Fig. S2). Rats performed $295 \pm 86$ trials per session and we recorded $27 \pm 15$ pPCx neurons per session. Recording sites were confirmed by post hoc histological analysis of tetrode lesion sites (Fig. S2).

To investigate how odor identity and their delivery location are encoded by pPCx ensembles, we examined neural responses during the trial initiation period when rats sampled the odor stimulus. Figure 2, A to $\mathbf{D}$ shows an example session in which 30 neurons were simultaneously recorded. As expected, pPCx neurons were selective for odor identity (Fig. 2, A 
and $\mathbf{C})$. However, we found that individual neurons also displayed mixed selectivity for both odor identity and the location where the odor was sampled (Fig. 2, A and D). Strikingly, some neurons even fired selectively with respect to location but not odor identity (Fig. 2, A and B). In fact, across the entire set of $\mathrm{pPCx}$ recordings, we found that more $\mathrm{pPCx}$ neurons were selective for location than for odor identity (40\% vs. $29 \%$, Fig. $2 \mathrm{E})$, and the fraction of neurons that were selective for location and not odor identity was higher than the fraction showing odor selectivity alone (24\% vs. $13 \%$, Fig. 2E). The fraction of mixed selectivity neurons which were jointly selective to both odor and location (16\%) was greater than predicted chance (chance level: $11.6 \%$, binomial proportions test, $\mathrm{p}<0.05)$, indicating potential associative mechanisms for odor and location representations in pPCx. In these analyses of selectivity and responsivity, we aligned pPCx spike times to odor onset time. Similar results were obtained when we aligned spike times to first inhalation after odor onset (data not shown). Beyond location and odor identity, PPCx population was not significantly selective for other task variables such as egocentric actions (left versus right turn) or water reward (correct versus error) (Fig. S4).

To control for the possibility that the spatially-restricted firing of pPCx neurons reflected the influence of stray odors on the maze (potentially deposited by the rat (28), each recording session was split into two blocks, separated by a 15-20 min interval during which the rat was removed and the maze and ports were thoroughly cleaned with disinfectant and an enzymatic cleaner (Fig. 2J, see Methods). We found that spatial firing rates of individual neurons across the two blocks within a session were stable across locations (Fig. 2, K to M, Fig. S3). We quantified the stability of location representation between the two blocks by calculating the pairwise Pearson's correlation coefficient for population activity across different locations in these two blocks (Fig. 2M, left). Values along the diagonal band in the correlation coefficient matrix represent the similarity of the $\mathrm{pPCx}$ population response to the same location in two 
recording blocks. The correlation coefficient values were significantly higher along the diagonal than off-diagonal (r_diag $=0.78 \pm 0.08, r_{-}$offdiag $=0.58 \pm 0.09$, Wilcoxon rank sum test, $p<$ 0.01), indicating that location representations were stable between blocks. Similar results were obtained when we examined all recorded pPCx neurons (Fig. 2M, right. r_diag $=0.81 \pm 0.02$, r_offdiag $=0.56 \pm 0.02$, Wilcoxon rank sum test, $p<0.001)$. Together, these findings show that uncontrolled odor cues on the maze are unlikely to account for the pPCx location representations observed. To further test whether the observed differential spatial activation of neurons might be the result of other non-spatial factors, we looked for systematic biases in behavior and neural responses that might result from the non-uniform availability of odors across the maze. However, no such biases were observed for individual rats or recording sessions (Fig. 2F and $H$, also see Fig. 1E and F).

We computed the Pearson's correlation coefficients between pairs of odor and location response vectors for the 284 odor-selective and 395 location-selective neurons (Fig. 3A, similar results were obtained using the entire recorded population, Fig. S5). Surprisingly, correlation coefficients for location pairs were significantly lower than for odor pairs (Fig. 3, B and C, p < 0.001). Therefore, not only were more pPCx neurons selective across locations than odors (Fig. 2E), the pPCx population representation for different locations was also more distinct than for different odors. We next used linear classifiers (support vector machines, SVMs) to quantify how well an ideal observer could decode odor identity or port location by observing firing rates of pPCx neurons. Using a pseudo-population, pooling all recorded neurons across sessions, we found that near perfect decoding of location (90.2\%) could be achieved using approximately 250 pPCx neurons, whereas more than 600 neurons were required for maximum classification accuracy for odors (accuracy $=85.1 \%$ for 725 neurons) (Fig. 3D, left). Therefore, activity of pPCx ensembles appeared to be better at predicting port location over odor identity. Maximum 
odor decoding accuracy for CA1 neurons recorded in the same task was worse than pPCx for the same number of neurons (for 91 neurons, CA1 accuracy $=34 \%, p P C x$ accuracy $=46 \%$, see also Fig. S5D for CA1 population odor decoding across time). In contrast, fewer CA1 neurons were required to successfully decode the same locations (Fig. 3D, right, 90\% for 46 CA1 neurons), consistent with the prominent spatial information in CA1 populations. The relatively high number of $\mathrm{pPCx}$ neurons required to reach maximum classification accuracy for odors also suggests that pPCx could be functionally distinct from anterior PCx (aPCx), where neurons encode odors with high fidelity $(6,7)$.

Next, to understand the temporal evolution of odor and location representations, we performed a decoding analysis in time using only simultaneously recorded pPCx populations, which also accounts for potential noise correlations (29) (Fig. 3E; cross-validated, chance is $25 \%, n=33$ sessions; $30 \pm 13$ neurons/session). As expected, odor identity decoding only rose above chance after odor onset. Surprisingly, location decoding accuracy had a very different time course that rose before odor onset (Fig. 3E). This reflects the fact that odor valves were only opened upon port entry (rats reached port locations on average X sec before odor onset, Fig. S1D), but more importantly, this reveals that decoding of location was not dependent on olfactory sensory drive. In fact, the accuracy of location decoding was similar in pre- versus post-odor onset periods (Fig. 3F, blue dots, accuracy_pre $=43.0 \pm 1.6$, accuracy_post $=47.4 \pm$ 1.8).

One possibility is that this spatial information reflects task-related contextual signals only occurring around odor sampling. Alternatively, however, this spatial information could be something akin to a spatial map, in which case, we would expect to see spatial representation that is consistent and stable across behavioral states in the task. To examine this, we analyzed the stability of location representation of neurons around different behavioral events within the 
task and during the inter-trial interval (ITI), where no odors or rewards were delivered (Fig. 4A, initiation, goal, and ITI epochs). Individual neurons displayed similar firing patterns across the maze and across these different behavioral epochs (Fig. 4B). At the population level, we quantified the stability of $\mathrm{PPCx}$ location representation by obtaining the similarity matrices for population location vectors across behavioral epochs. For both pPCx and CA1, location was similarly well encoded between initiation and goal epochs, as reflected by the diagonal axis of higher correlation coefficients in the similarity matrices (Fig. 4C). As evaluated by this method, the stability of pPCx spatial representations was similar to that of area CA1 (Fig. 4E, r_ppc_same $=0.63 \pm 0.04, r \_p p c \_d i f f=0.28 \pm 0.03, r \_$ca1_same $=0.64 \pm 0.13, r \_c a 1 \_$diff $=$ $0.17 \pm 0.13$, Wilcoxon rank sum test, $p<0.05)$. Spatial stability also held between the initiation epoch and ITI (Fig. 4C and D, r_ppc_same $=0.59 \pm 0.03$, r_ppc_diff $=0.31 \pm 0.03$, r_ca1_same $=0.54 \pm 0.12, r_{-}$ca1_diff $=0.12 \pm 0.06$, Wilcoxon rank sum test, $\left.p<0.05\right)$. We trained a linear classifier using firing rates from the initiation epoch and tested whether it was able to decode location from neural activity during goal or ITI epochs. Location classification was successful above chance and comparable to the cross-validated decoding during initiation (Fig. 4E, pPCx: $n=27$ sessions, 8-53 neurons/session. CA1: $n=12$ sessions, 2-13 neurons/session). Together, these results demonstrate that spatial representation in pPCx can be dissociated from olfactory sensory drive and are stable and persistent across temporal and behavioral epochs.

What could be the functional relationship between the spatial signals we observed in pPCx and the hippocampus? To address this, we used coherence analysis (30). We found that firing patterns of pPCx neurons, on average, were synchronous with respiration, as reflected by a continuous band of coherence that peaked in frequency during sniffing associated with odor sampling (Fig. 5Bi). As expected, spiking of CA1 population was coherent with hippocampal local field potential (CA1-LFP) (31) (Fig. 5Bii). We quantified the coherence of spiking in 
individual pPCx neurons with sniffing or CA1-LFP, based on their decoding performance for odor or location (Fig. 5C). We found that the accuracy of location-coding pPCx neurons was selectively correlated with the strength of spike-to-CA1-LFP coherence, but not correlated to the strength of spike-sniff coherence (Fig. 5Dii). In contrast, the accuracy of odor-coding neurons was robustly correlated with spike-sniff coherence (Fig. 5Di), indicating that they were more coupled to feedforward sensory drive. This result was reproduced when we computed the difference in coherence for the best odor-decoding vs best location-decoding neurons (Fig. S6). Our findings here indicate that pPCx neurons carrying spatial information are preferentially and functionally connected to the hippocampal navigation circuit.

While the behavioral and neural basis of olfactory search behaviors such as plume-tracking and chemical gradient sensing have been studied (32-35), the manner in which rodents use odors as landmarks in the environment, in combination with their spatial memory system in order to guide navigation, has received much less attention $(32,36,37)$. In this study, we examined the function of rodent olfactory cortex while rats used odor cues for spatial navigation, and found that pPCx contained information not only about odor identity but also carried robust information about the animal's location throughout different behavioral contexts. In contrast, simultaneously recorded CA1 neurons largely encoded space and not odor identity. While pPCx odor-place map could be derived from and reciprocally interact with hippocampal spatial maps (12), we posit that local learning and plasticity mechanisms within pPCx may also contribute to the spatial representations in pPCx. Indeed, consistent with an underlying associative mechanism for pPCx odor-place maps, the fraction of joint odor-location selective pPCx neurons is higher than chance-level prediction. This conjunctive representation of odor identity and location in pPCx has the necessary features to support navigation based on the use of odor 
locations as spatial landmarks, and could allow animals to anchor the presence of different odors within a spatial reference frame to guide navigation.

The prevalent use of olfactory learning in the investigation of hippocampal function (38) reflects the close functional relationship between olfactory and spatial memory systems. Lesion and perturbation studies have have been largely consistent with the canonical functional division between olfactory and hippocampal-entorhinal systems in attributing odor associations to olfactory cortex, and spatial memory to the hippocampal network (19, 20, 39-42). However, these previous studies have not examined activity in olfactory cortex during spatial behaviors. Here, the existence in pPCx of odor-selective neurons that fire in a spatially restricted manner not only sheds new light on our understanding of olfactory cortex function during behavior, but also speaks to the fundamental question of how sensory information is anchored within memory systems and combined with cognitive maps in the brain to guide flexible behaviors.

\section{Authorship Contributions:}

The project was originally conceptualized by C.P. and Z.M. and further developed in collaboration with N.B. The behavioral paradigm was developed and designed by C.P., N.B, and Z.M. Task-related hardware was developed and constructed by N.B. and C.P. Task-related software was developed and implemented by N.B. Animal training, behavior data collection, and behavioral data analysis was performed by C.P., and N.B. Neural recordings were performed by C.P. Neural data analysis was performed by C.P. and G.A. The manuscript was written by C.P., G.A., Z.M. and edited and reviewed by all authors. Supplementary materials and methods were written and provided by C.P., G.A and N.B. 


\section{References:}

1. E. C. Tolman, Cognitive maps in rats and men. Psychol. Rev. 55, 189 (1948).

2. J. S. Taube, R. U. Muller, J. B. J. Ranck, Head-direction cells recorded from the postsubiculum in freely moving rats. I. Description and quantitative analysis. J. Neurosci. 10, 420-435 (1990).

3. J. S. Taube, R. U. Muller, J. B. J. Ranck, Head-direction cells recorded from the postsubiculum in freely moving rats. II. Effects of environmental manipulations. J. Neurosci. 10, 436-447 (1990).

4. J. O'Keefe, J. Dostrovsky, The hippocampus as a spatial map. Preliminary evidence from unit activity in the freely-moving rat. Brain Res. 34, 171-175 (1971).

5. M. Fyhn, S. Molden, M. P. Witter, E. I. Moser, M.-B. Moser, Spatial representation in the entorhinal cortex. Science. 305, 1258-1264 (2004).

6. K. Miura, Z. F. Mainen, N. Uchida, Odor representations in olfactory cortex: distributed rate coding and decorrelated population activity. Neuron. 74, 1087-1098 (2012).

7. K. A. Bolding, K. M. Franks, Complementary codes for odor identity and intensity in olfactory cortex. Elife. 6, e22630 (2017).

8. D. C. Barnes, R. D. Hofacer, A. R. Zaman, R. L. Rennaker, D. A. Wilson, Olfactory perceptual stability and discrimination. Nat. Neurosci. 11, 1378-1380 (2008).

9. K. Miyamichi, F. Amat, F. Moussavi, C. Wang, I. Wickersham, N. R. Wall, H. Taniguchi, B. Tasic, Z. J. Huang, Z. He, E. M. Callaway, M. A. Horowitz, L. Luo, Cortical representations of olfactory input by trans-synaptic tracing. Nature. 472, 191-196 (2011).

10. D. L. Sosulski, M. L. Bloom, T. Cutforth, R. Axel, S. R. Datta, Distinct representations of olfactory information in different cortical centres. Nature. 472, 213-216 (2011).

11. L. B. Haberly, Parallel-distributed processing in olfactory cortex: new insights from morphological and physiological analysis of neuronal circuitry. Chem. Senses. 26, 551-576 (2001).

12. L. Wang, Z. Zhang, J. Chen, A. Manyande, R. Haddad, Q. Liu, F. Zu, Cell-type-specific whole-brain direct inputs to the anterior and posterior piriform cortex. Front. Neural Circuits (2020) (available at http://repository.uwl.ac.uk/id/eprint/6698/).

13. A. Diodato, M. Ruinart de Brimont, Y. S. Yim, N. Derian, S. Perrin, J. Pouch, D. Klatzmann, S. Garel, G. B. Choi, A. Fleischmann, Molecular signatures of neural connectivity in the olfactory cortex. Nat. Commun. 7, 12238 (11/2016).

14. K. L. Agster, R. D. Burwell, Cortical efferents of the perirhinal, postrhinal, and entorhinal cortices of the rat. Hippocampus. 19, 1159-1186 (2009).

15. F. C. Leitner, S. Melzer, H. Lütcke, R. Pinna, P. H. Seeburg, F. Helmchen, H. Monyer, Spatially segregated feedforward and feedback neurons support differential odor 
processing in the lateral entorhinal cortex. Nat. Neurosci. 19, 935-944 (2016).

16. M. E. Hasselmo, C. Linster, in Models of Cortical Circuits, P. S. Ulinski, E. G. Jones, A. Peters, Eds. (Springer US, Boston, MA, 1999), pp. 525-560.

17. L. B. Haberly, J. M. Bower, Olfactory cortex: model circuit for study of associative memory? Trends Neurosci. 12, 258-264 (1989).

18. J. M. Bower, M. A. Wilson, (1988), pp. 114-126.

19. C. Meissner-Bernard, Y. Dembitskaya, L. Venance, A. Fleischmann, Encoding of Odor Fear Memories in the Mouse Olfactory Cortex. Curr. Biol. 29, 367-380.e4 (2019).

20. G. B. Choi, D. D. Stettler, B. R. Kallman, S. T. Bhaskar, A. Fleischmann, R. Axel, Driving opposing behaviors with ensembles of piriform neurons. Cell. 146, 1004-1015 (2011).

21. C. Zelano, A. Mohanty, J. A. Gottfried, Olfactory predictive codes and stimulus templates in piriform cortex. Neuron. 72, 178-187 (2011).

22. D. J. Calu, M. R. Roesch, T. A. Stalnaker, G. Schoenbaum, Associative encoding in posterior piriform cortex during odor discrimination and reversal learning. Cereb. Cortex. 17, 1342-1349 (2007).

23. A. Hagiwara, S. K. Pal, T. F. Sato, M. Wienisch, V. N. Murthy, Optophysiological analysis of associational circuits in the olfactory cortex. Front. Neural Circuits. 6, 18 (2012).

24. J. D. Howard, J. Plailly, M. Grueschow, J.-D. Haynes, J. A. Gottfried, Odor quality coding and categorization in human posterior piriform cortex. Nat. Neurosci. 12, 932-938 (2009).

25. F. Restle, Discrimination of cues in mazes: A resolution of the " place-vs.-response" question. Psychol. Rev. 64, 217 (1957).

26. P. Lavenex, F. Schenk, Integration of olfactory information in a spatial representation enabling accurate arm choice in the radial arm maze. Learn. Mem. 2, 299-319 (1996).

27. N. Uchida, Z. F. Mainen, Speed and accuracy of olfactory discrimination in the rat. Nat. Neurosci. 6, 1224-1229 (2003).

28. D. G. Wallace, B. Gorny, I. Q. Whishaw, Rats can track odors, other rats, and themselves: implications for the study of spatial behavior. Behav. Brain Res. 131, 185-192 (2002).

29. B. B. Averbeck, P. E. Latham, A. Pouget, Neural correlations, population coding and computation. Nat. Rev. Neurosci. 7, 358-366 (2006).

30. P. Mitra, H. Bokil, Observed Brain Dynamics (Oxford University Press, USA, 2007).

31. J. O'Keefe, M. L. Recce, Phase relationship between hippocampal place units and the EEG theta rhythm. Hippocampus. 3, 317-330 (1993).

32. K. L. Baker, M. Dickinson, T. M. Findley, D. H. Gire, M. Louis, M. P. Suver, J. V. Verhagen, K. I. Nagel, M. C. Smear, Algorithms for Olfactory Search across Species. J. Neurosci. 38, 
9383-9389 (2018).

33. A. G. Khan, M. Sarangi, U. S. Bhalla, Rats track odour trails accurately using a multi-layered strategy with near-optimal sampling. Nat. Commun. 3, 703 (2012).

34. K. C. Catania, Stereo and serial sniffing guide navigation to an odour source in a mammal. Nat. Commun. 4, 1441 (2013).

35. R. T. Cardé, M. A. Willis, Navigational strategies used by insects to find distant, wind-borne sources of odor. J. Chem. Ecol. 34, 854-866 (2008).

36. L. F. Jacobs, From chemotaxis to the cognitive map: the function of olfaction. Proc. Natl. Acad. Sci. U. S. A. 109 Suppl 1, 10693-10700 (2012).

37. D. H. Gire, V. Kapoor, A. Arrighi-Allisan, A. Seminara, V. N. Murthy, Mice Develop Efficient Strategies for Foraging and Navigation Using Complex Natural Stimuli. Curr. Biol. 26, 1261-1273 (2016).

38. H. Eichenbaum, Using olfaction to study memory. Ann. N. Y. Acad. Sci. 855, 657-669 (1998).

39. R. P. Kesner, M. R. Hunsaker, W. Ziegler, The role of the dorsal and ventral hippocampus in olfactory working memory. Neurobiol. Learn. Mem. 96, 361-366 (2011).

40. K. M. Igarashi, L. Lu, L. L. Colgin, M.-B. Moser, E. I. Moser, Coordination of entorhinal-hippocampal ensemble activity during associative learning. Nature. 510, 143-147 (2014).

41. Y. Li, J. Xu, Y. Liu, J. Zhu, N. Liu, W. Zeng, N. Huang, M. J. Rasch, H. Jiang, X. Gu, X. Li, M. Luo, C. Li, J. Teng, J. Chen, S. Zeng, L. Lin, X. Zhang, A distinct entorhinal cortex to hippocampal CA1 direct circuit for olfactory associative learning. Nat. Neurosci. 20, 559-570 (2017).

42. U. Stäubli, G. Ivy, G. Lynch, Hippocampal denervation causes rapid forgetting of olfactory information in rats. Proc. Natl. Acad. Sci. U. S. A. 81, 5885-5887 (1984). 


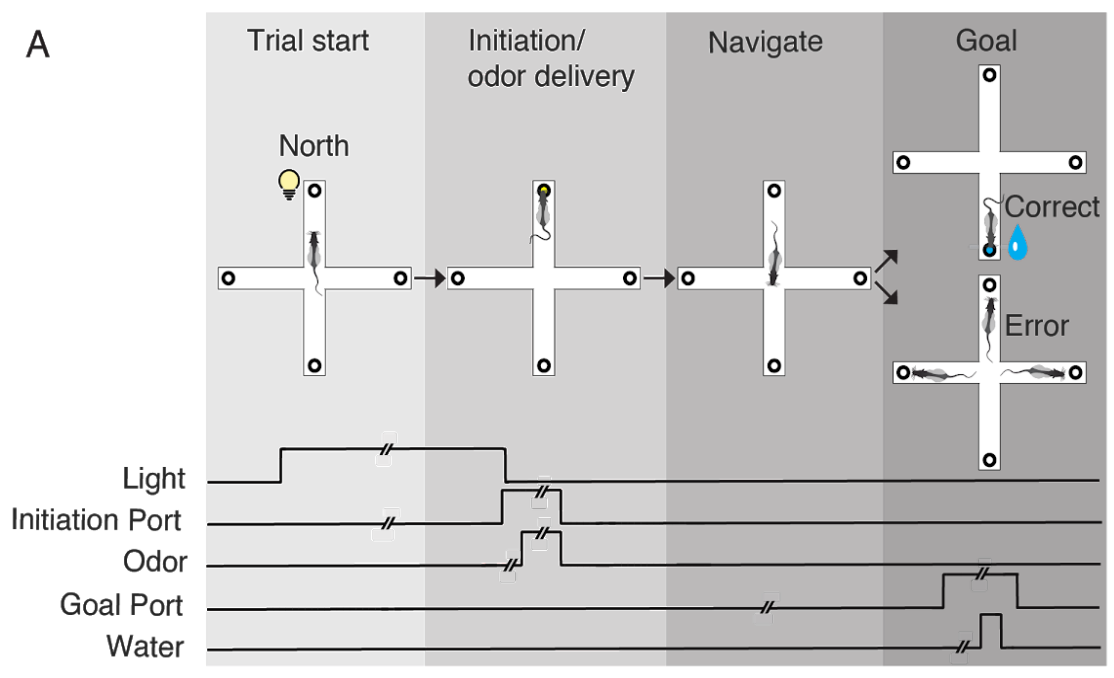

B

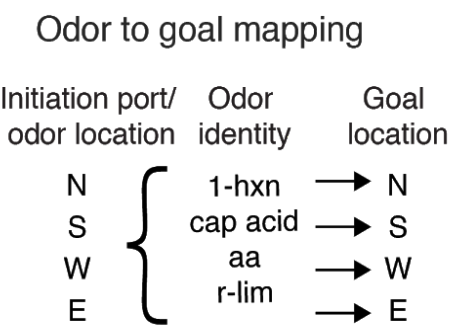

Trial trajectories

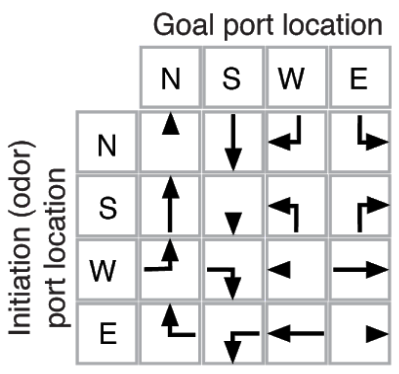

C

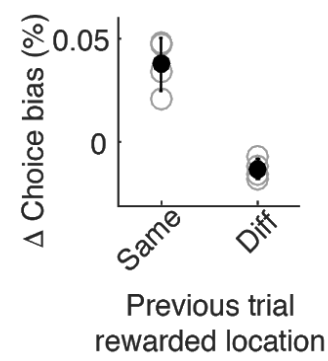

E

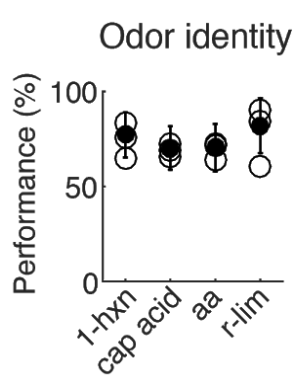

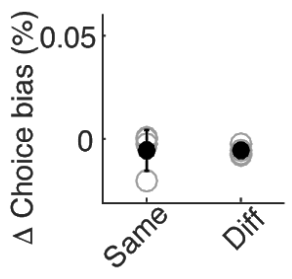

Previous trial rewarded action

$\mathrm{F}$ Port location

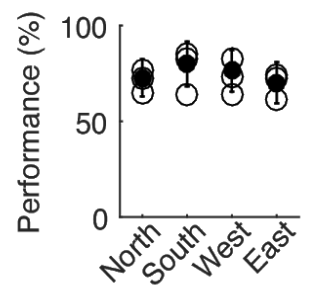

Figure 1. Odor-cued spatial choice task. (A) Task structure. Rats behave on elevated plus-maze with a nose port at the end of each arm. Each port can act as either an initiation (odor) port or a goal (reward) port. The rat initiates a trial by following a light cue to poke into a nose port to receive an (1 out of 4) odor stimulus. After a navigation period, the rat must poke into one of the four possible ports (North, South, West, or East port) to retrieve water reward. In a trial, an odor can be presented at any one of the four possible initiation ports. (B) Odor to goal location contingencies. Odors can be presented at one of four possible initiation (odor) ports, but each odor is associated with a fixed goal (reward) location, resulting in a total of 16 different trial trajectories. (C) Reward at a particular location biases the choice probability of rats towards the same location in the following trial (mean \pm S.D., $n=4$ locations, 6 rats, $\sim 40,000$ trials, see Supplementary Materials and Methods). Behavior of rats were positively biased for choosing the same location as previously rewarded location. (D) Reward for a particular action does not bias the choice probability for action in the following trial. Analysis similar as $C$ ( $n=4$ actions). (E) Behavioral performance of rats was similar across all four odor identities $(n=3$ 
implanted rats). (F) Behavioral performance of rats was similar across all four initiation (odor) port locations ( $n=3$ implanted rats).

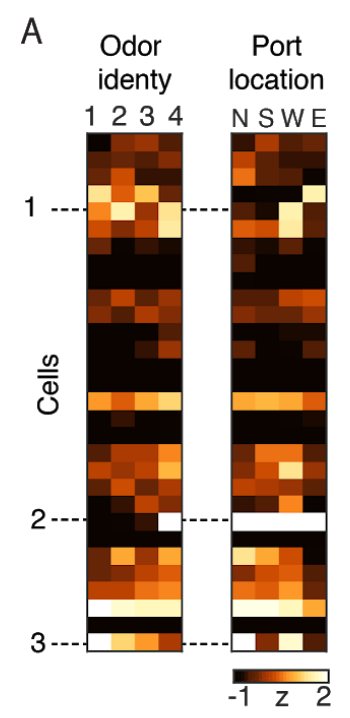

$\mathrm{E}$

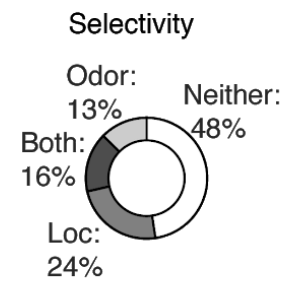

J

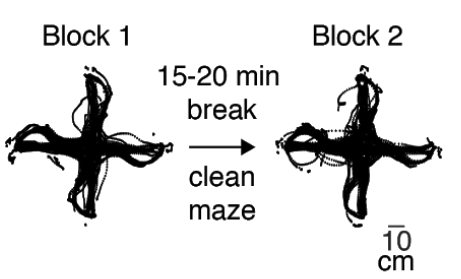

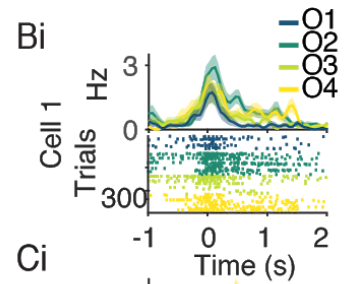

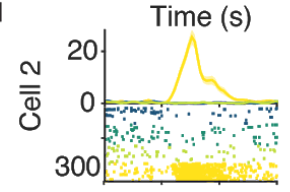

Di

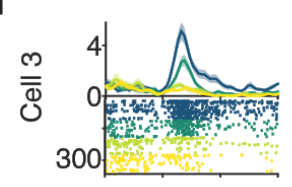

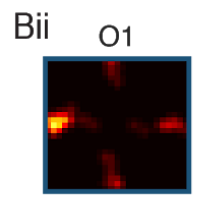

Cii

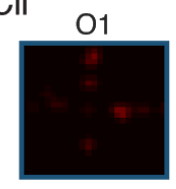

Dii
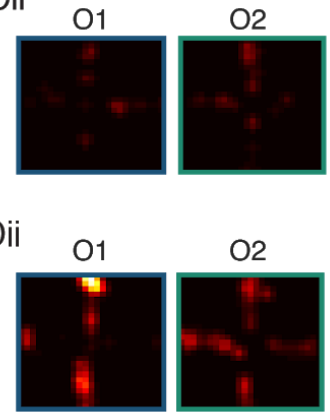
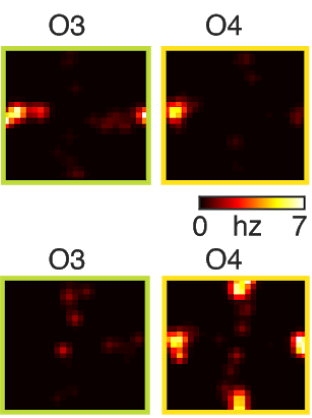

O3

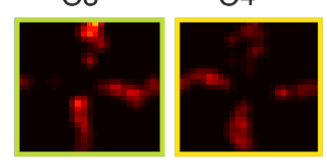

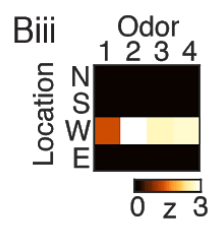
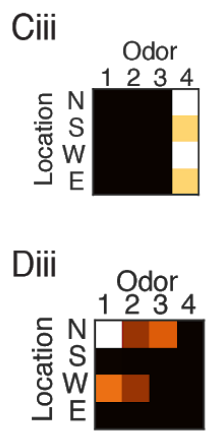

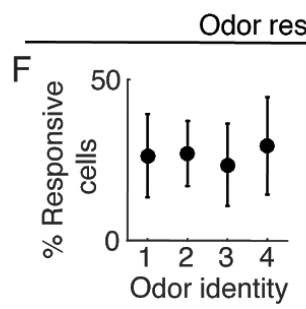

K Block 1 Block 2 NSWE NSWE
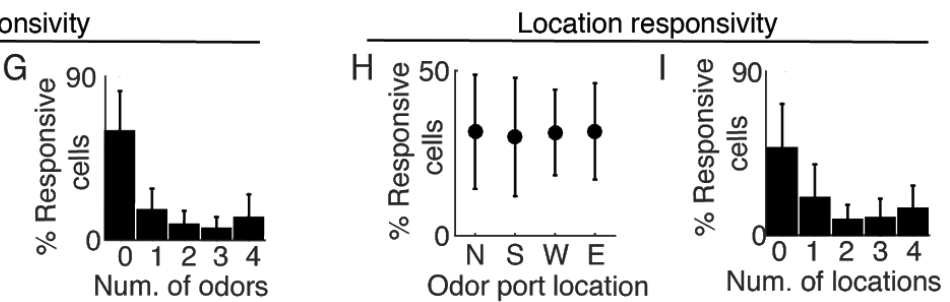

$\mathrm{M}$

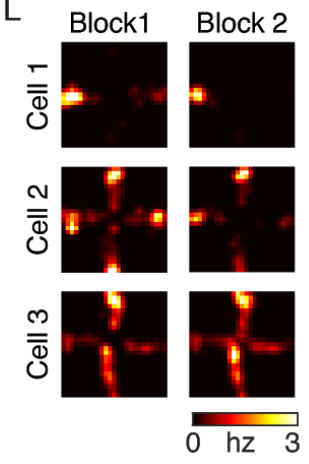

\section{All sessions}

Block 1
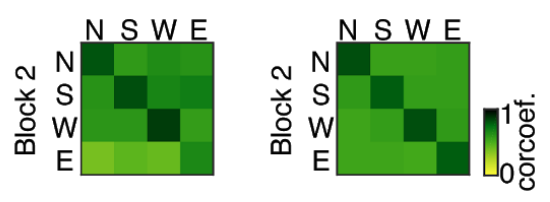

Figure 2. pPCx neurons are selective to odors and locations. (A-D) One example recording session of simultaneously recorded pPCx population ( $n=30$ cells). (A) Z-scored mean firing rate of neurons during odor sampling across odors and locations, show that neurons were differentially activated by odor and across port locations. Time window for mean firing rate was 1 second after odor onset. (B-D) 3 example cells from the session in (A). (Bi, $\mathbf{C i}, \mathbf{D i})$ Peri-event time histogram (PETH, top) and raster plots (bottom) reveal odor selectivity. Spike times were aligned to odor onset. (Bii, Cii, Dii) Firing rate heat map for trials in which different odors were sampled reveal location selectivity for odors. (Biii, Ciii, Diii) Z-scored responses for all odors and locations. (E) Selectivity of neurons for odors, locations, or both (See Methods). 29\% of pPCx neurons were odor selective, $40 \%$ were location selective, and $16 \%$ were selective for both odors and locations. (F) Fraction of recorded cells in a session that was 
activated by each odor ( $n=44$ sessions, mean \pm S.D.). Activation was defined as a significant difference in mean firing rate for 1 second after odor onset time compared to a 2 second baseline immediately preceding odor onset. Wilcoxon rank-sum test, $p<0.05$, corrected for multiple comparisons. Results were not significantly different when spikes were aligned to the first inhalation after odor onset instead of odor onset time. (G) Histogram of number of odors that activate cells in a session. (H) Fraction of recorded population in a session that was activated at each port location ( $n=44$ sessions). Activation was defined as a significant difference in mean firing rate for 1 second after initiation (odor) port poke-in to a 2 second baseline immediately preceding poke-in. Wilcoxon rank-sum test, $p<0.05$, corrected for multiple comparisons. (I) Histogram of the number of locations that activate cells in a session. ( $\mathbf{J}$ ) Rat position on the maze during two blocks within the same recording session. (K) Z-scored mean firing rate of neurons across blocks. (L) Heat map of firing rate for three example cells (same as cells in B-D) on the maze in two blocks. (M, left) Similarity matrix obtained by computing the pairwise Pearson's correlation coefficients between locations population response vectors for blocks 1 and 2 of example session shown in (A-F). Coefficients along the diagonal band are significantly higher than off-diagonal (r_diag $=0.78 \pm 0.08, r$ _offdiag $=0.58 \pm 0.09$, Wilcoxon rank sum test, $p<0.01$ ), representing stability of location representation across blocks. ( $\mathbf{M}$, right). Same analysis as in the left panel but for all pPCx neurons recorded $\left(r \_\right.$diag $=0.81 \pm 0.02, r \_$offdiag $=0.56 \pm 0.02$, Wilcoxon rank sum test $, p<0.001, n=44$ sessions, 995 neurons). 


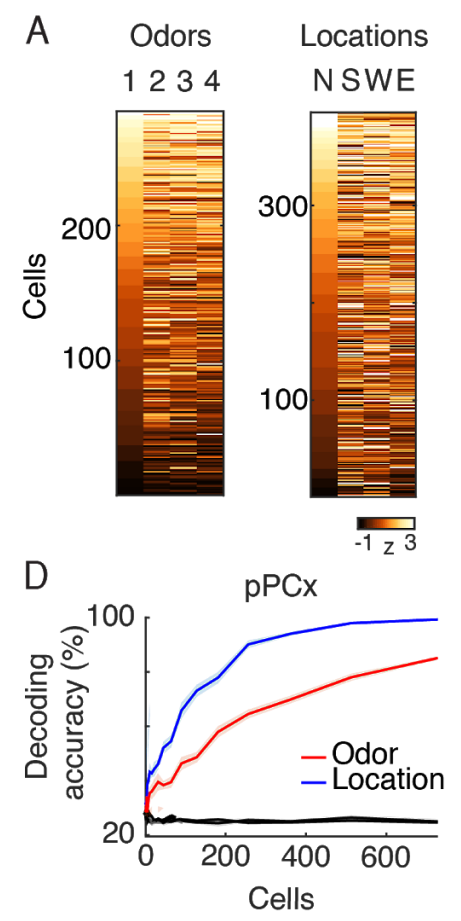

B C

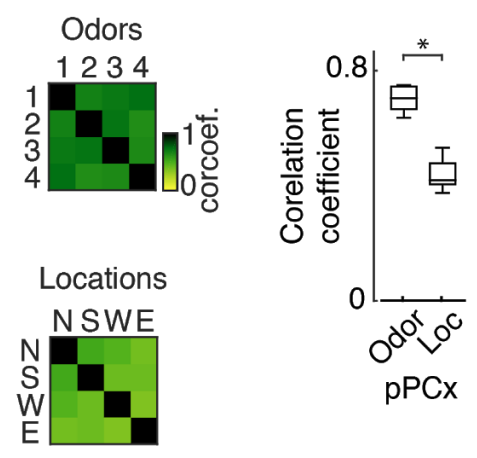

$E$
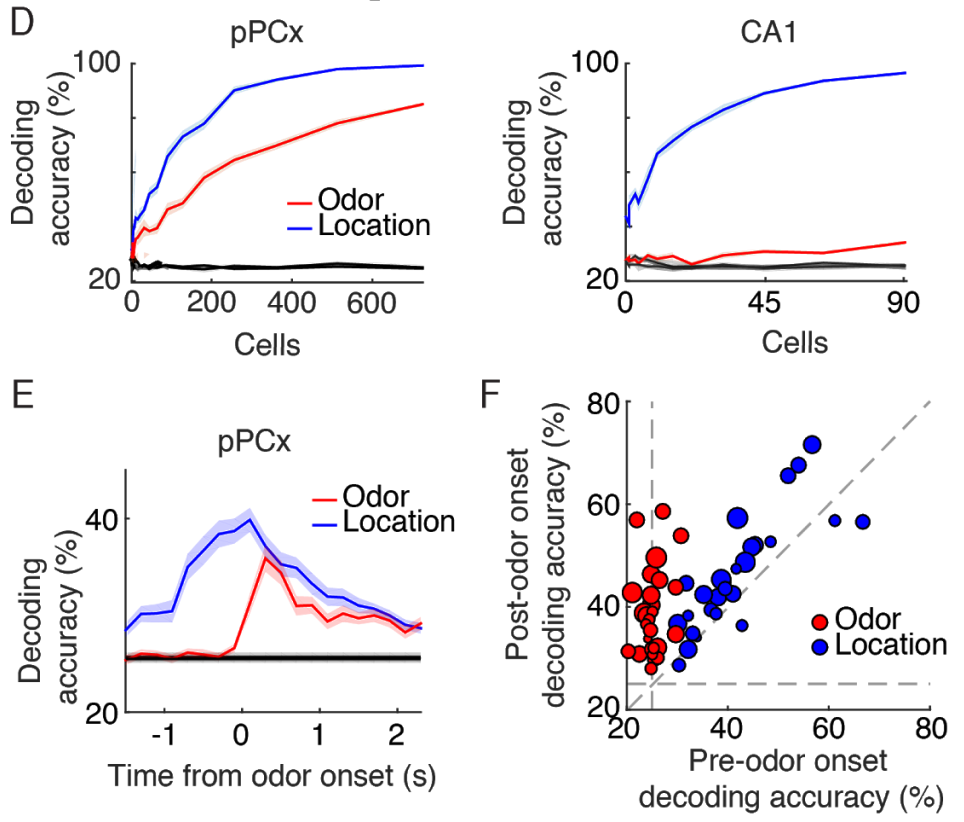

Figure 3. Odor and location decoded from single sessions of pPCx recordings. (A, left) Z-scored firing rate for odor selective neurons ( $n=284$ cells) within odor sampling duration for all four odors. Cells were sorted from lowest to highest response for odor 1. (A, right) Z-scored firing rate for location selective neurons ( $n=395$ cells) within odor sampling duration at all four port locations. Cells were sorted from lowest to highest response for north port. (B, top) Similarity matrix obtained by computing the pairwise Pearson's correlation coefficients between all population response vectors for odors in (a, left). Squares along the diagonal band are 1 representing autocorrelation. (B, bottom) Similarity matrix obtained by computing the pairwise Pearson's correlation coefficients between all population response vectors for locations in (a, right). Squares along the diagonal band are 1 representing autocorrelation. (C) pPCx population correlation coefficients for odor and locations in B excluding autocorrelation coefficients represented by diagonal band. r_ppc_odor $=0.69 \pm 0.04, \quad$ r_ppc_loc $=0.43 \pm 0.05$. (Wilcoxon rank sum test, $\mathrm{p}<0.001$ ). Box plot central mark: median, box edges: 25th,75th percentiles, whiskers: range of data points). (D) pPCx (left) and CA1 (right) pseudo-population decoding accuracy for odors (red) and locations (blue). Lines are means of 10 subsampled pseudo-populations. (E) Decoding accuracy of simultaneously recorded pPCx populations (Mean \pm S.E.M., $n=27$ sessions; 30 \pm 13 neurons/session). (F) Decoding accuracy of simultaneously recorded pPCx populations before 
and after odor stimulation. Each dot corresponds to a single session, with dot size indicating number of simultaneously recorded neurons $(n=27$ sessions, 8-53 neurons/session, accuracy_pre $=43.0 \pm 1.6$, accuracy_post $=47.4 \pm 1.8)$.

A

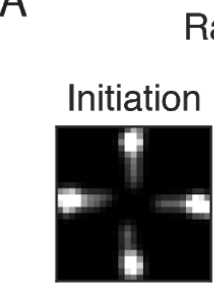

Rat occupancy

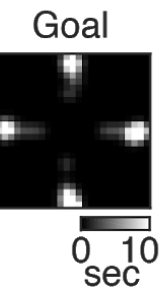

ITI

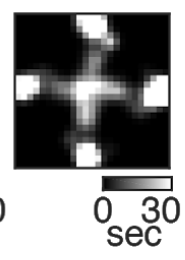

B

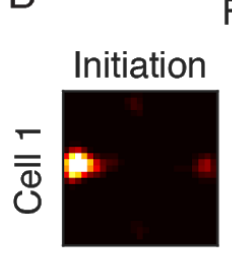

Firing rates
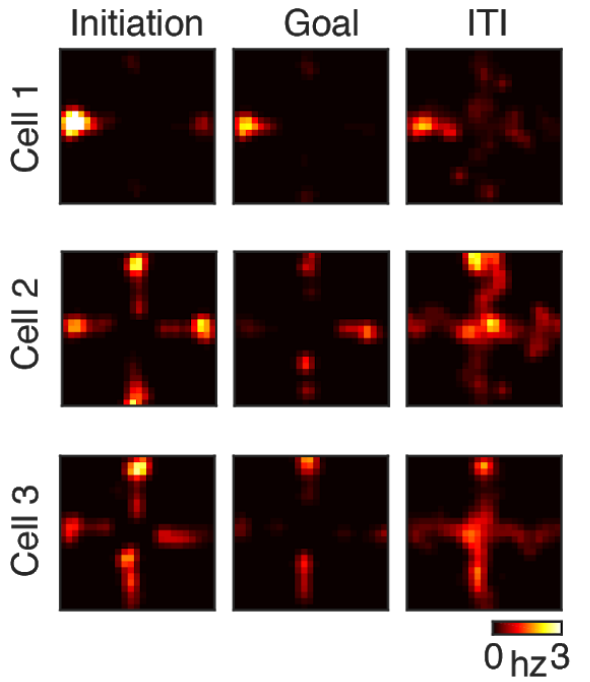

$\mathrm{Ci}$

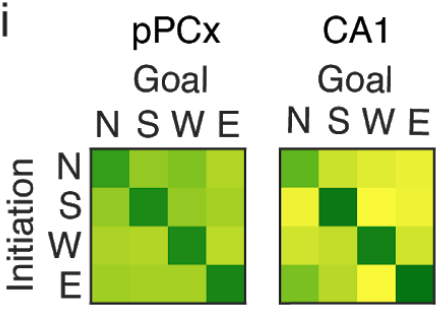

Di
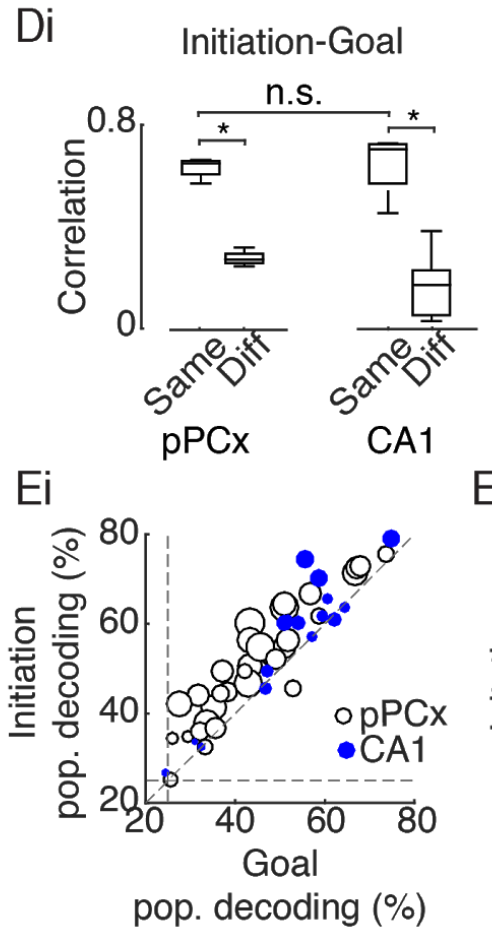

Cii pPCx

CA1

ITI

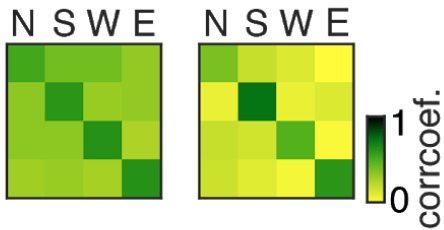

Dii

Initiation-ITI

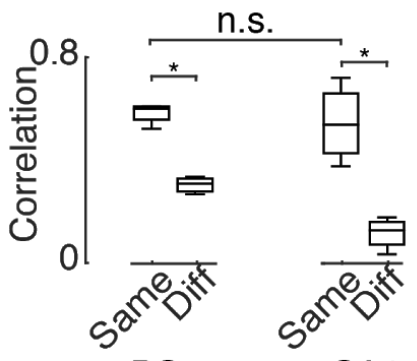

Eii

CA1

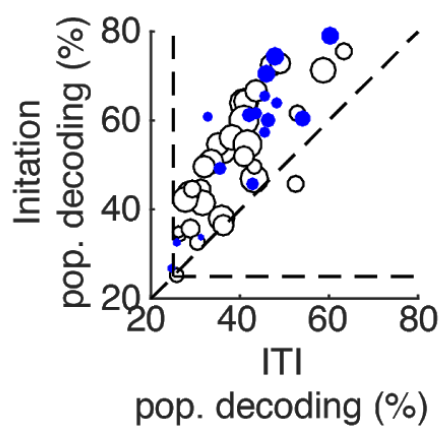

Figure 4. Persistence of pPCx spatial representation across behavioral epochs. (A) Occupancy of rat on maze in an example session during three behavioral epochs for all trials in session. For each trial, initiation epoch is $1 \mathrm{sec}$ window around odor port entry. Goal epoch is $1 \mathrm{sec}$ window around goal port entry. Inter-trial-interval (ITI) is enforced to be 4-6 sec between trials. (B) Heat map of firing rate normalized for occupancy in 3 example cells (same cells as Fig $2 \mathrm{f}-\mathrm{h}$ ) during different behavioral epochs. (Ci) Similarity matrices for pPCx (left) and CA1 (right) spatial representation between initiation and goal epochs. Correlation is performed on population response vectors at port locations for all location selective neurons ( $n=395$ pPCx cells, 103 CA1 cells). (Cii) Similarity matrices for pPCx (left) and CA1 (right) spatial representation between initiation and ITI epochs. (Di) Summary of correlation coefficients for initiation and goal epochs for pPCx and CA1 location population vectors. Higher correlation coefficients at the same versus different refer to diagonal versus off-diagonal values in (c) (r_ppc_same $=0.63 \pm 0.04, r \_p p c \_d i f f=0.28 \pm 0.03, r$ rca1_same $=0.64 \pm 0.13, r$ _ca1_diff $=0.17 \pm$ 0.13 , Wilcoxon rank sum test, $p<0.05$ ). (Dii) Summary of correlation coefficients for initiation and ITI epochs for pPCx and CA1 location population vectors (r_ppc_same $=0.59 \pm 0.03, r \_p p c \_d i f f=0.31 \pm$ 
0.03, r_ca1_same $=0.54 \pm 0.12, r \_c a 1$ diff $=0.12 \pm 0.06$, Wilcoxon rank sum test, $\left.p<0.05\right)$. (Ei) Linear SVM classifier trained on neural responses during initiation epoch successfully decode location from neural responses during the goal epochs. (Cross-validated, chance is $25 \%$. pPCx: $n=27$ sessions, $8-53$ cells/session; CA1: $n=12$ sessions, 2-13 cells/session). (Eii) Linear SVM classifier trained on responses during initiation epoch successfully decode location from neural activity during ITIs. 
A
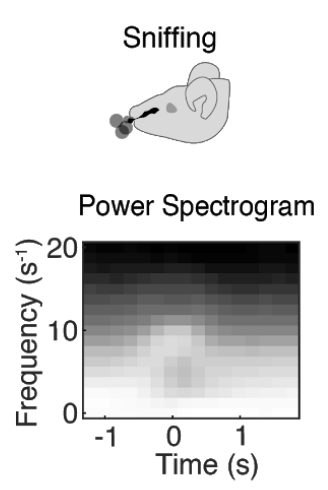

$\mathrm{Bi}$
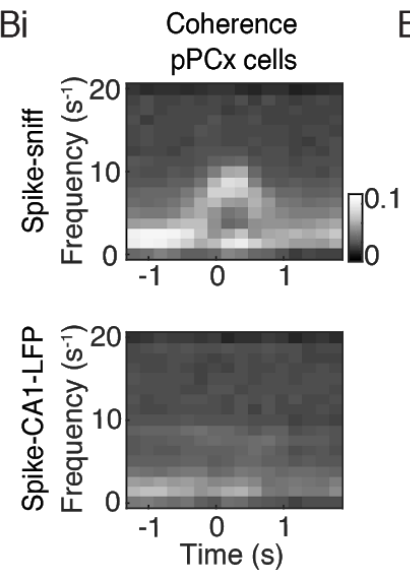

CA1-LFP

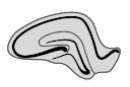

Power Spectrogram

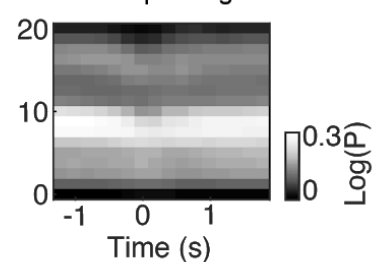

Bii Coherence
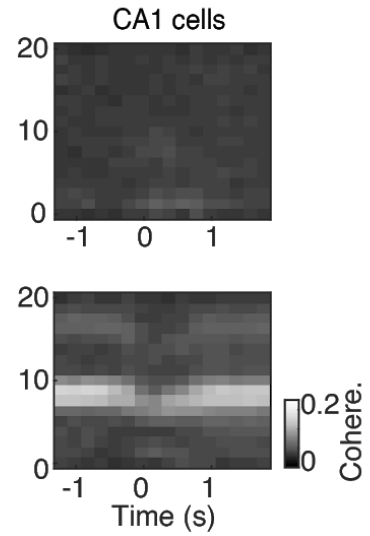

$\mathrm{Ci}$

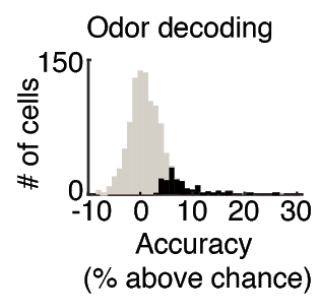

Di
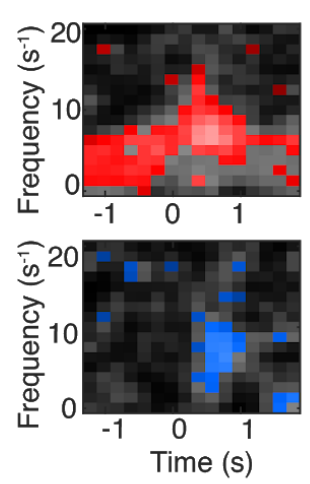

E

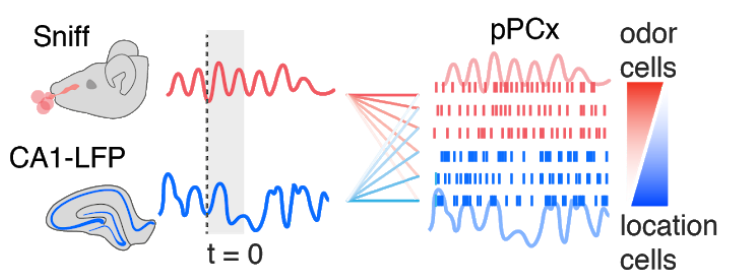

Figure 5. Location decoding PPCx neurons are coupled to CA1-LFP and not sniffing. (A) Average power spectrum of sniffing (left) and CA1 LFP (right) aligned to odor onset time. Brightness are log(Power). (Bi) Average coherence of pPCx spikes to sniff (top) and CA1-LFP (bottom) aligned to odor onset time. (Bii) Average coherence of CA1 spikes to sniffing (top) and CA1-LFP (bottom) aligned to odor onset time. (C) Decoding performance of individual pPCx neurons for odor (Ci) and location (Cii). Significance was defined as accuracy greater than the 95th percentile of classifiers trained on shuffled labels. (Di) Correlation between odor decoding performance and coherence of spikes to sniffing (top) and CA1-LFP (bottom). Colored pixels indicate frequency-time bins in which decoding performance was significantly correlated with sniffing (red) and CA1-LFP (blue). (Dii) Similar analysis as (Di) but for location decoding accuracy. (E) Schematic of preferential coupling between odor and locations cells to sniffing and CA1-LFP, respectively. 


\section{Supplementary Figures S1-6}

A

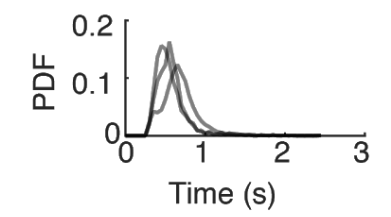

C

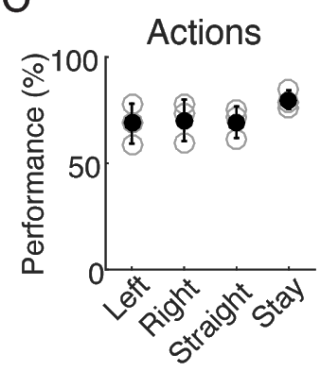

$E$

$2 \mathrm{~Hz}$ sniffing on maze

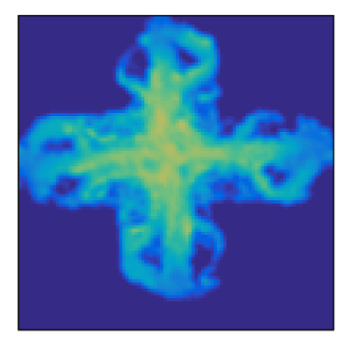

$\mathrm{F}$

Sniffing synchronized to poke-in

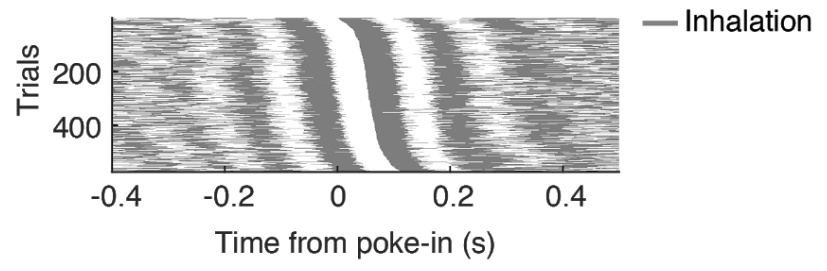

Figure S1. Odor sampling behavior in task. (A) Odor sampling duration in 3 recorded rats. Minimum of $150 \mathrm{~ms}$ of odor sampling is enforced. (B) Navigation duration in 3 recorded rats, as defined by time between initiation (odor) port poke-out and goal port poke-in. Goal ports were only active (reward available) after a 2 second delay after rat left odor port. This was to discourage rats from staying at the same location. (C) Performance for trials in which odor sampling location is the same as goal location (stay) is higher than other actions ( $n=3$ rats, 44 sessions; performance left: $68 \pm 0.09$; right: $70 \pm 0.1$; straight: $69 \pm 0.07$; stay: $78 \pm 0.04$ ). (D) Distance to initiation port location aligned to odor onset time $(\mathrm{n}$ = 3 rats, 44 sessions). (E) Sniffing behavior on maze in one example session. Heat map of basal sniffing behavior (2 Hz power) and high frequency active sniffing behavior $(7-10 \mathrm{~Hz})$ on maze. (F) Sniff phase was time-locked to initiation (odor) port poke-in. Gray color is inhalation, white is exhalation. 
A

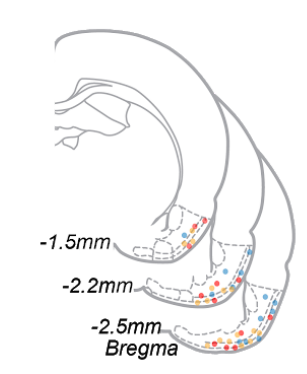

B

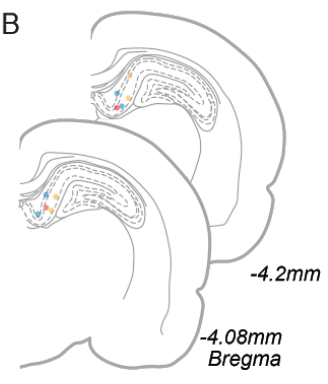

C

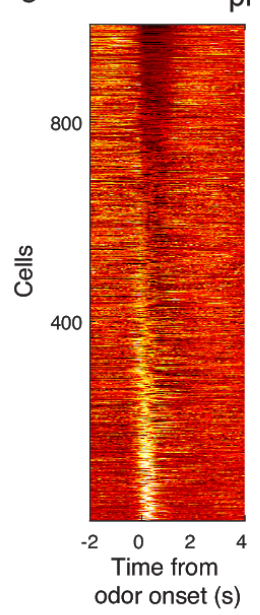

D

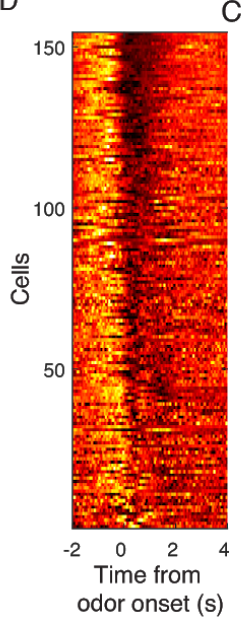

E

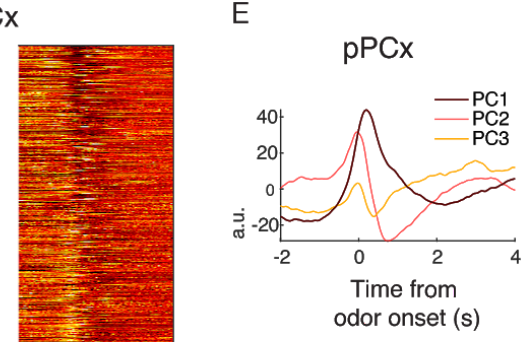

CA1

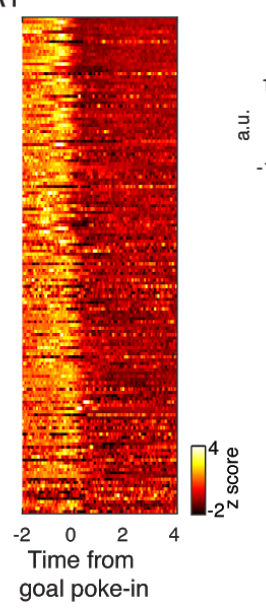

G

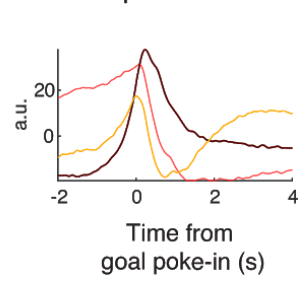

$\mathrm{H}$

CA1
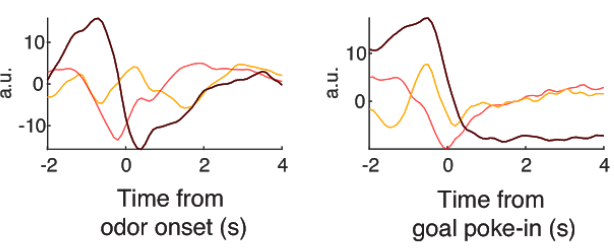

Figure S2. (A, B) Tetrode lesion sites for 3 recorded rats in pPCx and CA1. (C-H) pPCx and CA1 population response summary for aligned to odor onset and goal poke-in. (C,D) Summary PETH for all pPCx $(n=995)$ and CA1 $(n=154)$ neurons recorded. Spike timing was aligned to odor onset (left) and goal poke-in (right). Neurons were sorted based on their activity during 1 second time window after odor onset in both left and right panels. For each neuron, the mean z-scored rate during a 2 second time window prior to alignment time point was subtracted from the entire PETH. (E-H) First two principal components for pPCx and CA1 population responses aligned to odor onset and goal poke-in show distinct time courses for pPCx and CA1. 


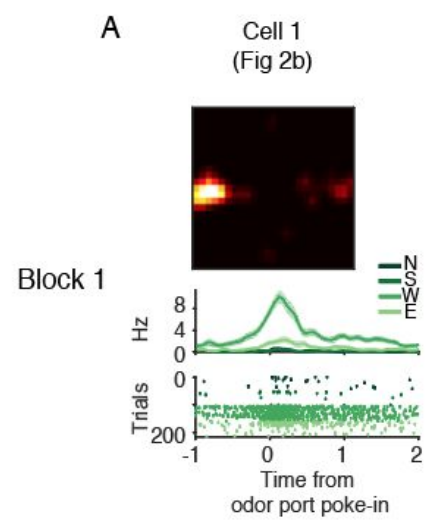

B $\quad \begin{gathered}\text { Cell } 2 \\ \text { (Fig 2c) }\end{gathered}$
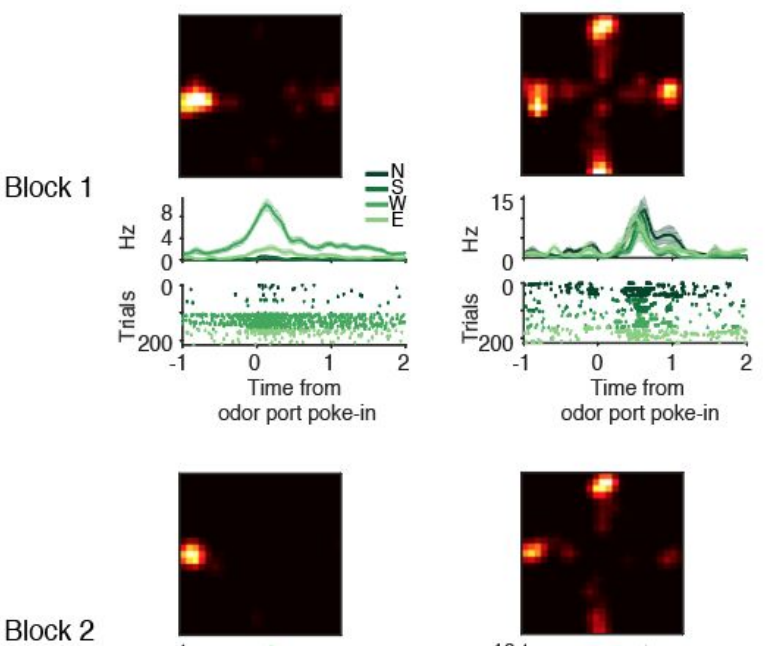

N.
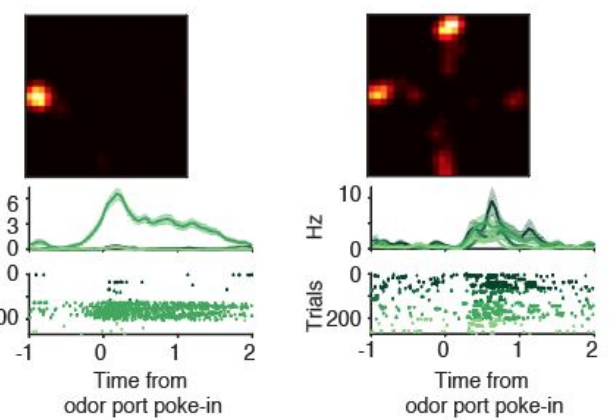

C Cell 3

(Fig 2d)
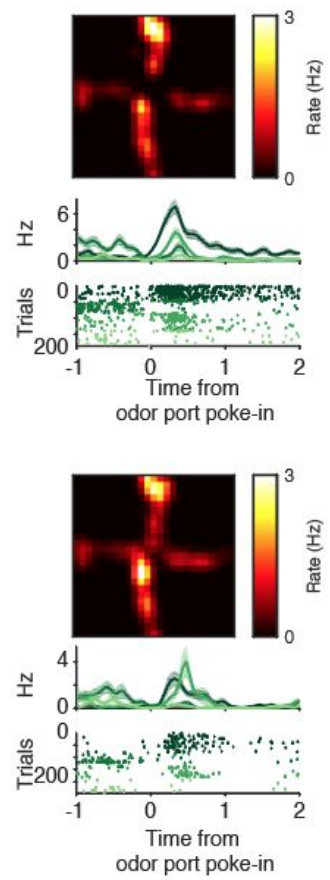

Figure S3. Stability of location representation in pPCx. Firing rate heat map, PETH, and raster plots for 3 example cells (same as cells shown in Fig. 2B-D, L) showing stability of location selectivity of individual neurons. PETH and raster plots were aligned to odor port pok-in and sorted by port locations. 
pPCx

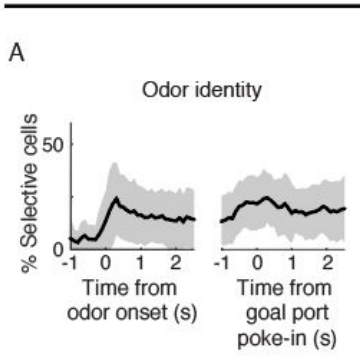

C

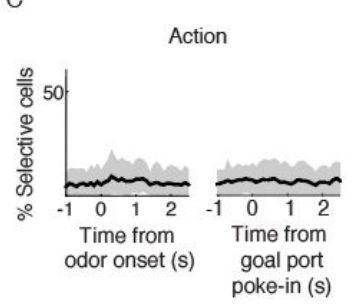

B

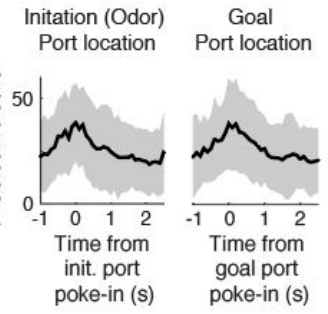

D

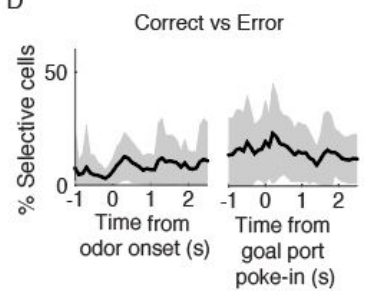

CA1

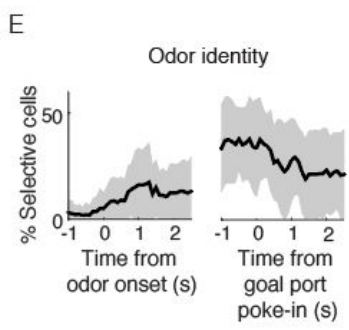

$\mathrm{G}$

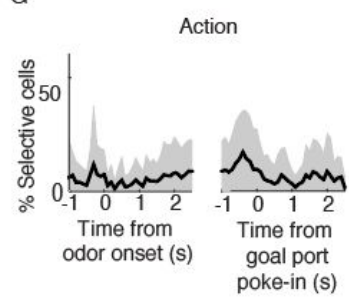

F Initation (Odor) Goal Port location Port location

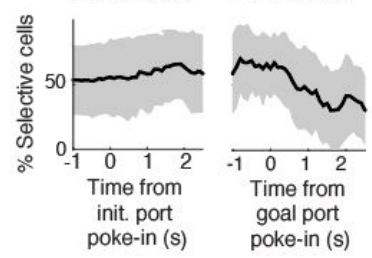

H

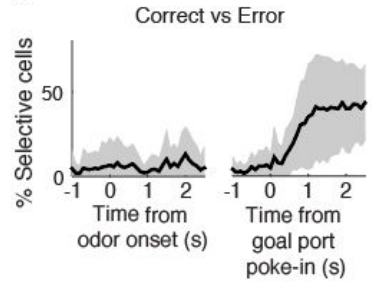

Figure S4. Time course of population selectivity of pPCx and CA1 for task related variables. (A) pPCx selectivity for identity of odor stimulus. (B) pPCx selectivity for current port location (initiation or goal port) aligned to initiation or goal port poke-in time. (C) pPCx selectivity for left versus right action choice during navigation period. (D) pPCx selectivity for correct (rewarded) versus error (un-rewarded) trials. Selectivity for variables was evaluated by comparing firing rates of neurons across conditions within a 0.25 second sliding time window using ANOVA-test, $p<0.05$. $n=44$ session, 30 \pm 13 neurons/session neurons/session. (E-H) CA1 selectivity quantified similar to (A-D). $n=25$ sessions, 2-26 neurons/session. 

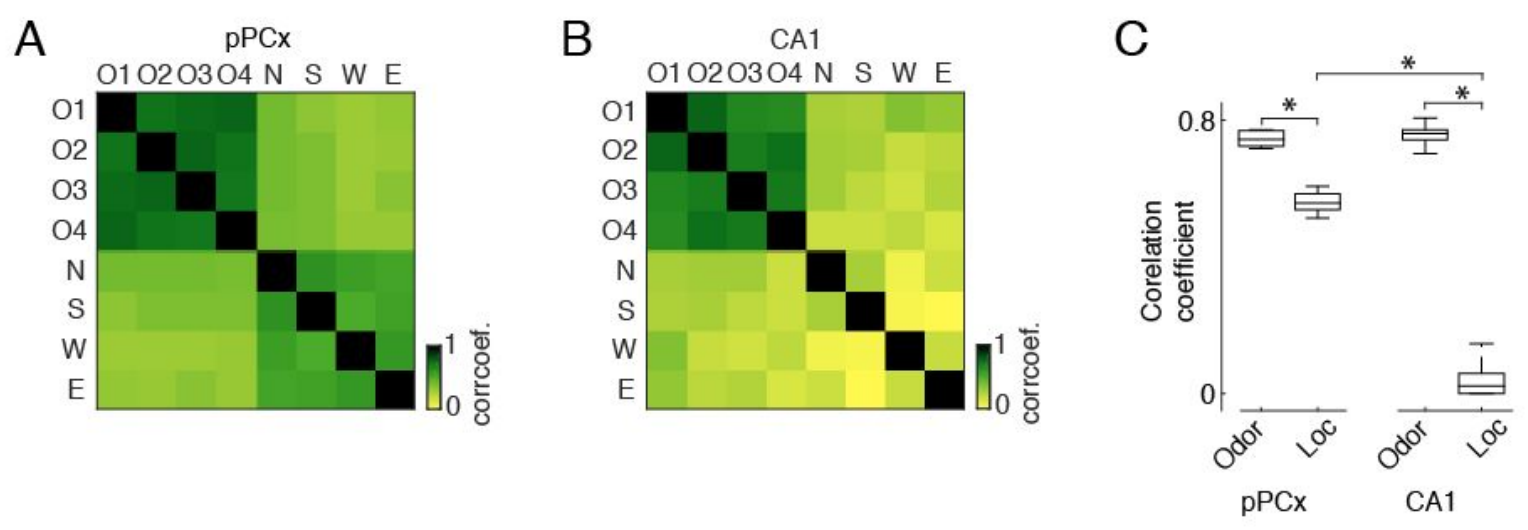

Figure S5. (A) Full similarity matrix of correlation coefficients of all odor and location population vector pairs for all recorded pPCx neurons ( $n=995$ neurons). (B) Full similarity matrix of correlation coefficients of all odor and location population vector pairs for all recorded CA1 neurons ( $n=154$ cells). (C) Off-diagonal correlation coefficients odors and locations show that pPCx location representations are more dissimilar from each other than pPCx odor representations. CA1 location representations are more dissimilar from each other than CA1 odor representations as well as pPCx location representations (Wilcoxon rank sum test, $p<0.05$ ). 


\section{$\Delta$ coherence}
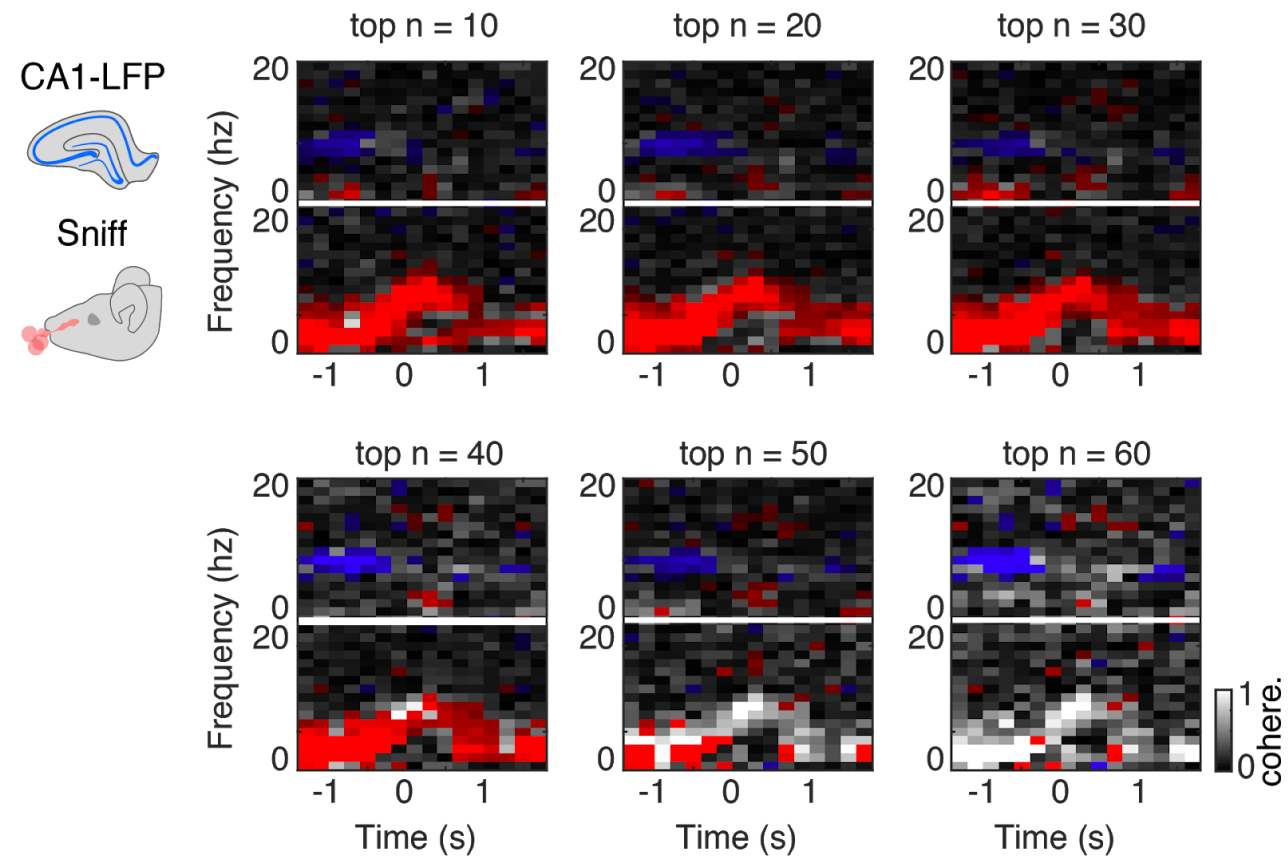

Location cells sign. more coherent than odor cells

Odor cells sign. more

coherent than location cells

Fig. S6. Relationship between pPCx decoding performance and coherence to sniffing or CA1-LFP. Mean difference in spike-sniff coherence between the 'top n' odor and location decoding pPCx neurons.Top panels of each 'top n' analysis show spike-sniff coherence, while bottom panels show spikes-to-CA1-LFP coherence. Gray scale is coherence. Red pixels indicate frequency-time bins in which spikes from odor decoding cells are significantly more coherent than spikes from location decoding neurons, while blue pixels indicate the bins in which location cells are more significantly coherent than odor cells. 


\section{Materials and Methods}

All experiments and procedures were approved by the Champalimaud Foundation Bioethics Committee and the Portuguese National Authority for Animal Health, Direcção-Geral de Alimentação Veterinária (DGAV).

\section{Animal Subjects}

A total of 6 male adult Long-Evans hooded rats (4-6 months and weighing 450-550 g) were used in experiments. All 6 rats were used for behavioral analysis (Fig. 1C and D), 3 rats were implanted for neural recordings (Fig. 2 to 5 and all supplementary figures).

\section{Odor-cued spatial navigation task}

Rats were trained and tested on an odor-cued four-alternative spatial choice task where water was used as a reward based on tasks previously described (1). Here, rats were trained on an elevated plus maze with open arms and a $25 \mathrm{~mm}$ ledge. At the end of each arm there was one nose port which could deliver a light cue, odors, or water reward (modified from Island Motion)(2). Each trial followed the structure of initiation/odor delivery, navigation, and reward. A trial begins with a light cue indicating the initiation port for that trial. Rats initiated a trial by nose-poking at the port indicated by a light cue. This triggered the delivery of an odor with a (uniform) random delay of $0.1-0.25 \mathrm{~s}$ (Fig. 1A). Initiation port location was pseudo-randomized within 10 trial blocks and balanced across all port locations. Rats were trained to stay in the odor sampling port for a minimum of $0.15 \mathrm{~s}$, after which they were free to leave (Fig. S1). Odor delivery was terminated as soon as the rat exited the odor port. Following poke-out of the initiation port, a minimum two second time delay for choice/navigation is enforced (Fig. S1) to discourage rats from their preference to stay at their current location to collect reward. After this delay period, water reward is available at the correct port location. Each odor was associated with one possible reward location (North, South, West or East). A poke in the correct location would yield a tone $(80 \mathrm{~ms} 3 \mathrm{KHz})$ and a $30 \mu \mathrm{l}$ water reward while a poke in an incorrect port would yield an error tone (80ms white noise burst). A 4 to 6 seconds (uniform distribution) inter-trial interval (ITI) period started after the delivery of the reward. Reward was available for correct choices for up to $10 \mathrm{~s}$ after the rat left the odor sampling port. Water was delivered with a random delay from entry into the goal port drawn from a uniform distribution of $0.5-1.0 \mathrm{~s}$. The task was designed and implemented using a RTLFSM and B-control as described previously (3). A Point Gray Flea3 1.3MP camera was used to track the rat's behavior. The Bonsai framework (4) was used to interface with the cameras.

\section{Odor stimuli}

Odor delivery was controlled by a custom made olfactometer (3). We used relatively low concentration of odorants (liquid dilution factor: 1:100 in mineral oil and further diluting 100 $\mathrm{ml} / \mathrm{min}$ odorized air in a total of $1000 \mathrm{ml} / \mathrm{min}$ clean air stream). All odor stimuli (1-Hexanol, Caproic Acid, R-Limonene, and Amyl Acetate) were randomly interleaved during a session.

\section{Training}

Rats had free access to food, but water was restricted to the training sessions and 5-10 additional minutes of free access per day. Rats were trained in $45 \mathrm{~min}$ sessions, twice a day, 5-7 days/week. Each training session was spaced 6-8 hours apart for motivation. To prevent over-training, in $20 \%$ of trials (pseudo-randomly interleaved), the correct choice is indicated by a light cue at the correct goal port (answer trials). In the remaining $80 \%$ of trials, all 4 possible goal ports are lit (question trials). A threshold of $75 \%$ correct for question trials (chance is $25 \%$ ) 
is taken as performance criterion. All behavioral and neural data analysis were restricted to question trials only.

\section{Microdrive implant}

After reaching an asymptotic performance in behavioral training, each rat was implanted with a custom-designed and $3 \mathrm{D}$ printed multielectrode drive (based on microdrives designed in the laboratory of Dr. Loren Frank, U.C.S.F) (PolyJetHD Blue, Stratasys Ltd.) with 24 independently moveable tetrodes (based on design from the laboratory of Dr. Loren M. Frank., UCSF). Tetrodes (Ni-Cr, California Fine Wire Company) were gold plated to reach an impedance of $250 \mathrm{k} \Omega$ at $1 \mathrm{kHz}$. Implanted recording drives had two cannulas that targeted both pPCx (19 tetrodes, cannula angled at 19 degrees away from the midline), and dorsal CA1 (5 tetrodes, vertical cannula). Cannulas were centered at the following coordinates: right pPC $-2.2 \mathrm{~mm}$ AP and $3.0 \mathrm{~mm} \mathrm{ML}$, right dorsal hippocampal CA1 (-4.2 mm AP and $2.0 \mathrm{~mm} \mathrm{ML})$. LEDs were attached to the microdrive and used for video tracking of rat position.

\section{Neural recordings}

After 10 days of post-operative care and recovery, rats were water restricted and trained in the same manner as the pre-surgery period. Tetrodes were adjusted every 2 days post-surgery to reach the target coordinate, guided by depth, LFP, and spiking patterns. Before starting recordings, animals were retrained to reach similar accuracy levels as those achieved before surgery $(>75 \%$ correct for question trials). Recording sessions were split into two $45 \mathrm{~min}$ behavioral blocks with a 15-20min rest period in between blocks to allow for rats to rest and consume food in a separate box. During this time, surfaces of the maze and port were cleaned thoroughly with enzymatic cleaner (Henry Schein Medical) and disinfectant (VirkonTM-S, and $70 \%$ ethanol). Electrical signals were amplified and recorded using the Cerebus data acquisition system (Blackrock Microsystems, Utah). Thresholded events were recorded at $20 \mathrm{kHz}$ (for spike sorting), continuous signal was recorded at $2 \mathrm{kHz}$ (for LFP). Tetrode depths were adjusted at the end of each session in order to sample an independent population of neurons across sessions. The locations of tetrode tips during each recording session were estimated based on their depth and histological examination based on electrolytic lesions and the visible tetrode tracks (Fig. S2). Rats performed 1 session per day, and a total of 44 recording sessions were obtained from 3 rats.

Recording and analysis of respiration pattern: To monitor sniffing, during drive implantation, a temperature sensor (custom T-type probe, 44ga., Physitemp Instruments) was implanted in one nostril, and respiration patterns were monitored as a temperature change in the nasal cavity as described previously (3). Signals from nasal thermocouple were amplified, filtered between 0.1 and $475 \mathrm{~Hz}$ and digitized at $2,000 \mathrm{~Hz}$. For analysis, voltage signals were further low-pass filtered $(<50 \mathrm{~Hz}$ ). Onset of inhalations and exhalations were identified as local maxima and minima of the signals semi-automatically using custom software.

\section{Histology}

To verify the ultimate location of the tetrodes, electrolytic lesions were produced after the final recording session (30 $\mu \mathrm{A}$ of cathodal current, $3 \mathrm{~s}$ ). Rats were deeply anesthetized with pentobarbital and perfused transcardially with $4 \%$ paraformaldehyde (wt/vol in PBS). The brain was sectioned at $50 \mu \mathrm{m}$ and stained with Cresyl violet solution to observe sites of electrolytic lesions (Fig. S2). 


\section{Data Analysis}

All data analysis was performed with custom-written software using MATLAB (Mathworks). No statistical methods were used to pre-determine sample sizes.

Behavioral bias analysis: Delta bias is the change in probability of choosing a particular location in the next trial as a consequence of the outcome of the current trial. We can express this as:

$$
\Delta \operatorname{Bias}\left(\operatorname{Loc}_{t-1}, \operatorname{Loc}_{t}, \operatorname{Loc}_{t+1}, R_{t}\right)=P\left(\operatorname{Loc}_{t+1} \mid \operatorname{Loc}_{t}, R_{t}\right)-P\left(\operatorname{Loc}_{t-1} \mid \operatorname{Loc}_{t}, R_{t}\right)
$$

Where $\mathbf{t}$ is the current trial; location Loc $\in\{$ North , South, West, East $\}$ and reward $\mathbf{R} \in\{0,1\}$. The Location bias is defined as the delta bias of repeating the same location and results from fixing the Location and conditioning the analysis to rewarded and non rewarded trials:

$$
\operatorname{LocBias}(R)=\sum_{l}^{L o c} \Delta \operatorname{Bias}(l, l, l, R)
$$

The same analysis was performed for actions by substituting Loc for Action $\in$ Left, Right, Forward, Stay\}.

Spike Sorting: Single units were isolated offline by manually clustering spike features derived from the waveforms using spike-sorting software provided by D. N. Hill, S. B. Mehta, and D. Kleinfeld (5). Single-units recorded on more than one session, as judged from the spike waveform and the firing pattern, were excluded from the analysis, but the results were not affected by the inclusion of all units. Recordings from outside the pPCx or CA1 were excluded from the analysis. We also excluded units with < 200 spikes in a given session. As in other cortical areas, neurons could be classified into two categories: wide-spiking (width $>0.2 \mathrm{~ms}$ ) and narrow-spiking neurons (width $<0.2 \mathrm{~ms}$ ). The width was determined as the time between a peak and a trough of the mean spike waveform. About $4 \%$ (39) of recorded neurons fell into the category of narrow-spiking neurons. Overall, pPC neurons had spontaneous firing rates of 2.79 $\pm 3.74 \mathrm{~Hz}$ (mean \pm S.D.). Spontaneous firing rates of wide-spiking neurons were significantly lower than those of narrow-spiking neurons $(2.23 \pm 2.49 \mathrm{~Hz} ; 16.41 \pm 3.43 \mathrm{~Hz}$; mean \pm S.D., respectively). Both categories of cells were included in the analyses but exclusion of narrow spike neurons did not affect the main conclusions.

Single neuron analysis: Odors: In order to obtain instantaneous firing rates (e.g. for Peri-event time histograms, PETHs), spike events were convolved with a Gaussian filter (S.D.: 25 ms) (Fig. 2B). To identify responses of individual neurons that were significantly changed with odor stimuli, we compared the firing rate of a 1 second time window after odor onset to a 2 second time window immediately prior to odor onset using the Wilcoxon rank sum test, $p<0.05$ (Fig. 2F and $\mathbf{G}$ ). To identify neurons that were odor selective (Fig. 2E), we compared the firing rate during a 1 second time window after odor onset across the four odor stimuli using an ANOVA-test, $p<0.05$. Locations: Responsiveness of individual neurons to port locations (Fig. $\mathbf{2 H}$ and I) was evaluated by comparing the firing rate of a 1 second time window after port poke-in with a 2 second time window immediately prior to poke-in at different port locations using the Wilcoxon rank sum test, $p<0.05$. Port location selectivity of individual neurons (Fig. 2E) was obtained from comparing activity across locations in the firing rate map (see 'Analysis of firing rate on maze' section below) using ANOVA-test.

Analysis of firing rate on maze: For firing rate heat map visualization (Fig. 2 B to D, I, Fig. 4B, Fig. S3), rat positions (tracked by LED on the microdrive) was cropped by the width and length of the maze ( 1 meter $\times 1$ meter) divided into $4 \times 4 \mathrm{~cm}$ bins. Mean firing rate for each position bin normalized by occupancy was obtained and then smoothed with a gaussian kernel (S.D.: $2 \mathrm{~cm})$. 
Firing rate heat maps for individual odors (Fig. 2Biii, Ciii, and Diii) were obtained sorting trials by odor identity, and obtaining the firing rate meap map for all concatenated trials of the same odor. Responses for each neuron used for location selectivity and correlation analysis was obtained from dividing the maze into $20 \times 20 \mathrm{~cm}$ bins. The mean z-scored firing rate for the position bin centered on each port was taken as location response.

Analysis of population activity across epochs (Fig. 4): Initiation and goal epochs were defined as 1 second time windows centered around initiation and goal port poke-in times, respectively. Inter-trial interval (ITI) epoch was defined as the 4-6 second time window enforced in between trials.

Population correlations (Fig. 2M, Fig. 3B and C, Fig. 4C and D, Fig. S5): Odor: Population odor response vectors consisted of mean z-scored firing rate of neurons within a 1 second time window after odor onset. Location: Population location response vectors consisted of mean z-scored firing rate on the maze as described above in the 'Analysis of firing rate on maze' section. Pearson's correlation coefficient were used for reported correlation coefficients. Qualitatively similar results were obtained using Spearman's rank correlation coefficient.

Pseudopopulation decoding (Fig. 3D): Subsets of neurons were sampled from across recording sessions. Population sizes were sampled at sqrt(2) increments up to the total number of available neurons leading to pseudopopulations of 2-725 (pPCx) and 2-91 (CA1). Neuronal responses were concatenated into a matrix, arranged so that each row contained responses to a particular (odor/location). Because different sessions contained different numbers of trials, a random subset of responses from each neuron was excluded so that the number of trials per category was consistent across neurons in the pseudopopulation. For each population size, the sampling procedure was repeated 10 times. Each neuron's mean firing rate was calculated during a one second time window after odor onset. Trials were labeled 1-4 according to the odor sampled (for odor decoding) or port location (for location decoding), and arranged into a $\mathrm{T}$ (\# trials)-element vector. The procedure was repeated with shuffled labels to determine chance-level performance (black line in Fig. 3D). Sessions with fewer than 400 trials per session was excluded, since it would lead to overall fewer usable trials in the entire pseudopopulation analysis.

Population decoding (Fig. 3E and F): For per-session decoding, neuronal firing rates were arranged into a matrix of size $\mathrm{T}$ (\# trials) $\mathrm{N}$ N (\# neurons). Trials were labeled 1-4 according to the odor sampled (for odor decoding) or port location (for location decoding), and arranged into a $\mathrm{T}$ (\# trials)-element vector. This matrix was normalized by taking the z-score of each column (so that each neuron's firing rate was standardized). The vector of labels and matrix of firing rates were fed into a linear, multi-class SVM classifier (libsvm for MATLAB, Software available at http://www.csie.ntu.edu.tw/ cjlin/libsvm (6)) using 5-fold cross validation. Chance-level classification was estimated by running the same classifier on shuffled labels. For pre-vs-post odor decoding (Fig. 3F), time windows of 1-second before and after initial poke-in were used. For decoder time courses (Fig. 3E), 200-ms non-overlapping windows were used. Data sessions with fewer than 8 pPCx neurons were excluded because it had substantially fewer simultaneously recorded cells.

Identification of CA1-LFP: For each rat, the 96-electrode LFP data was represented as a 96-dimensional matrix sampled at $40 \mathrm{~Hz}$. Each row of this matrix (representing a single 
electrode time series) was high-pass filtered using Matlab's zero-phase 'filtfilt' function, and a $2 \mathrm{~Hz}$, 2nd order Butterworth filter. The resulting matrix was truncated to include only the time window [-1.5s, 2s] around the time of all trial-initiating poke-ins taking place within a session. For a given rat, the procedure was repeated for all sessions, and the resulting matrices were concatenated into a single matrix. In summary, this pre-processing captured each animal's multi-electrode LFP during odor sampling and across sessions. SVD was applied to the resulting matrix, and the trial-averaged spectrograms (see below) of the top 10 components were visualized. To identify hippocampal theta, we looked for a component with power in the $\sim 8$ $\mathrm{Hz}$ band (7). For each rat, we picked the highest-variance component of this sort. Two additional lines of evidence suggested that we successfully identified theta: 1) theta power decreased around the time of poke-in, consistent with the observation that theta power decreases during periods of immobility (7); and 2) the components showed strong coherence with identified hippocampal spiking activity at $\sim 8 \mathrm{~Hz}$ (see Fig. 5B, bottom right panel) (8).

Spectral analysis: To compute power spectra and coherograms (Fig. 5A and B), the Chronux toolbox was used (http://chronux.org/) (9). Each neuron's coherence with sniffing and CA1-LFP was calculated as a function of time and frequency using the function cohgramcpb, using Fs = $40 \mathrm{~Hz}$, a sliding window of .5s with a stride of .2s, and taper set to [2 Hz, .5s, 1]. Similarly, the power spectrum for sniff and CA1-LFP was calculated using function specgram, with the same parameter settings. For this and subsequent analysis, data of 1 of 3 rats was excluded because it performed far fewer trials per session, leading to noisy estimates of coherence.

Single-cell decoding (Fig. 5C): For each neuron, the firing rate was sampled at $5 \mathrm{~Hz}$ over the interval [-1.5s, 2s] with respect to initial poke-in. This was repeated across all trials, resulting in an R (\# Trials) x T (\# Timesteps) matrix. Each column of the matrix (ie each time point) was z-scored. Corresponding R-element vectors containing labels for poke-in location and odor identity was defined. For each, a linear multi-class SVM decoder (using LIBSVM, see above) was trained to classify odor/location based on the firing rate time series. This procedure was repeated 1000 times for label-shuffled data to establish each neuron's baseline classification performance, which deviated from $25 \%$ because of non-uniform trial sampling in sessions. Finally, the mean chance-level performance was subtracted, and neurons whose classification accuracy exceeded the 95th percentile of the baseline classification accuracy were labeled as significant.

Relating single-cell decoding to coherence: Correlation between decoding accuracy and coherence (Fig. 5D, 6S): The coherograms of cells identified as significantly odor (location) coding (see above) were taken, and for each (time, frequency) value of the coherogram, the across-cell correlation between coherence and decoder was calculated. To identify (time, frequency) pairs with a significant association between decoding and coherence, the correlation was repeated 1,000x using shuffled decoder values, and $(T, F)$ pairs whose value exceeded the 95th percentile were colored red (for sniff) and blue (for CA1-LFP). Top ' $n$ ' cells analysis (Fig. 6S): The top 'n' odor- and location- decoding cells were taken from Fig. $5 \mathrm{C}$, and the difference of their mean coherograms was plotted. Significant (T,F) bins were identified as those whose values were significantly different between the top ' $n$ ' location and top ' $n$ ' odor cells, as indicated by a t-test (ttest2 in matlab). 


\section{References:}

1. N. Uchida, C. Poo, R. Haddad, Coding and transformations in the olfactory system. Annu. Rev. Neurosci. 37, 363-385 (2014).

2. G. Felsen, Z. F. Mainen, Neural substrates of sensory-guided locomotor decisions in the rat superior colliculus. Neuron. 60, 137-148 (2008).

3. N. Uchida, Z. F. Mainen, Speed and accuracy of olfactory discrimination in the rat. Nat. Neurosci. 6, 1224-1229 (2003).

4. G. Lopes, N. Bonacchi, J. Frazão, J. P. Neto, B. V. Atallah, S. Soares, L. Moreira, S. Matias, P. M. Itskov, P. A. Correia, R. E. Medina, L. Calcaterra, E. Dreosti, J. J. Paton, A. R. Kampff, Bonsai: an event-based framework for processing and controlling data streams. Front. Neuroinform. 9, 7 (2015).

5. D. N. Hill, S. B. Mehta, D. Kleinfeld, Quality metrics to accompany spike sorting of extracellular signals. J. Neurosci. 31, 8699-8705 (2011).

6. ChangChih-Chung, LinChih-Jen, LIBSVM. ACM Transactions on Intelligent Systems and Technology (TIST) (2011) (available at https://dl.acm.org/doi/10.1145/1961189.1961199).

7. G. R. Richard, A. Titiz, A. Tyler, G. L. Holmes, R. C. Scott, P.-P. Lenck-Santini, Speed modulation of hippocampal theta frequency correlates with spatial memory performance. Hippocampus. 23, 1269-1279 (2013).

8. J. O'Keefe, M. L. Recce, Phase relationship between hippocampal place units and the EEG theta rhythm. Hippocampus. 3, 317-330 (1993).

9. P. Mitra, H. Bokil, Observed Brain Dynamics (Oxford University Press, USA, 2007). 